\title{
Future risk of metabolic syndrome in women with a previous LGA delivery stratified by gestational glucose tolerance: a prospective cohort study
}

\author{
Heidi Hakkarainen ${ }^{1,2^{*}}$ (1) , Hanna Huopio ${ }^{3}$, Henna Cederberg ${ }^{4,5}$, Raimo Voutilainen ${ }^{3,6}$ and Seppo Heinonen ${ }^{7,8}$
}

\begin{abstract}
Background: Whether the delivery of a large-for-gestational-age (LGA) infant predicts future maternal metabolic syndrome (MetS) is not known. To this aim, we investigated the incidence of MetS and its components in women with or without a history of gestational diabetes mellitus (GDM) with a view to the birth weight of the offspring.

Methods: Eight hundred seventy six women treated for their pregnancies in Kuopio University Hospital in 19892009 underwent a follow-up study (mean follow-up time 7.3 (SD 5.1) years), of whom 489 women with GDM and 385 normoglycemic controls. The women were stratified into two groups according to the newborn's birth weight: 10-90th percentile (appropriate-for-gestational-age; AGA) $(n=662)$ and $>90$ th percentile (LGA) $(n=116)$. MetS and its components were evaluated in the follow-up study according to the International Diabetes Federation criteria.

Results: LGA vs. AGA delivery was associated with a higher incidence of MetS at follow-up in women with a background of GDM (54.4\% vs. 43.6\%), but not in women without GDM.

Conclusion: An LGA delivery in women with GDM is associated with a higher risk of future MetS and this group is optimal to study preventive measures for MetS. In contrast, an LGA delivery after a normoglycemic pregnancy was not associated with an increased future maternal MetS risk.
\end{abstract}

Keywords: Gestational diabetes mellitus, Metabolic syndrome, Large-for-gestational-age, Birth weight

\section{Background}

Gestational diabetes mellitus (GDM) increases the risk of obstetric complications, largely due to fetal overgrowth. In addition, GDM is associated with an increased risk of developing type 2 diabetes (T2DM) [1-3], metabolic syndrome (MetS) and cardiovascular diseases (CVD) [4-8] after the pregnancy. The key pathophysiological defects underlying the increased cardiometabolic morbidity after GDM pregnancy include chronic insulin resistance and impaired insulin secretion, together with visceral obesity, hypertension and dyslipidemia [9]. Disturbance in glucose metabolism is considered to be a major cause for a

\footnotetext{
* Correspondence: heidi.hakkarainen@kuh.fi

1 Department of Obstetrics and Gynecology, Kuopio University Hospital,

Puijonlaaksontie 2, P.O.B 100, 70029 KYS, Kuopio, Finland

${ }^{2}$ Institute of Clinical Medicine, School of Medicine, University of Eastern

Finland, P.O.B 1627, 70211 Kuopio, Finland

Full list of author information is available at the end of the article
}

large-for-gestational-age (LGA) delivery [10], albeit many environmental and genetic factors are also likely to play a role. In particular, maternal pre-pregnancy body mass index (BMI) and gestational weight gain have been shown to be independent determinants of the infant birth weight [11-13]. Women with pre-pregnancy overweight and obesity were at 1.5-fold and 2-fold increased risk of delivery of an LGA infant, respectively [12]. Furthermore, maternal metabolic factors including decreased high-density lipoprotein (HDL) cholesterol, increased triglycerides [14] and insulin have previously been shown to be independent determinants of fetal macrosomia [15]. In continuum, infant born LGA and exposed to an intrauterine environment of diabetes or maternal obesity have also been shown to be at an increased risk of developing MetS later in their lives [16].

We therefore hypothesized that a previous LGA delivery would be associated with an increased risk of

(c) The Author(s). 2018 Open Access This article is distributed under the terms of the Creative Commons Attribution 4.0 International License (http://creativecommons.org/licenses/by/4.0/), which permits unrestricted use, distribution, and 
incident MetS in the mother after the pregnancy. To this aim, we investigated the incidence of MetS and its components in women with and without GDM by groups of different birth size.

\section{Methods}

This hospital register-based cohort study included women whose pregnancies were treated in Kuopio University Hospital, Finland, in 1989-2009. Women who had the diagnosis of GDM and a random sample of normoglycemic women, both groups with completed oral glucose tolerance test (OGTT) during pregnancy, were contacted by a letter and invited for the study. A total of 489 women with GDM and 385 women with normal OGTT result during pregnancy attended the follow-up study. 1234 women did not reply or declined to participate in the study.

The women with and without GDM were classified based on the birth weight of the newborn: between 10-90th percentile (appropriate-for-gestational-age; AGA) $(n=662)$ and over 90th percentile (LGA) $(n=116)$. The women without GDM and delivery of an AGA infant served as a control group. In this study, LGA was defined as sex-specific birth weight for gestational age above the 90th percentile on the current Finnish newborn growth charts [17].

\section{Data collection during pregnancy}

In Finland, cost-free maternity care is offered to all pregnant women. The women considered to be at risk of GDM underwent 2-h OGTT (75 g glucose after overnight fasting) between the 24th and 28th weeks of gestation if one or more following factors were present: age over 40 years, BMI $\geq 25 \mathrm{~kg} / \mathrm{m}^{2}$, prior GDM or a history of a macrosomic delivery, glucosuria, suspected fetal macrosomia in the current pregnancy. The diagnostic criteria of GDM were as follows: until September 2001 the lower limits of abnormal fasting, 1-h and 2-h capillary whole-blood glucose 4.8, 10.0 and $8.7 \mathrm{mmol} / \mathrm{l}$ and since September 2001 the lower limits of fasting, 1-h and 2-h capillary plasma glucose 4.8, 11.2 and $9.9 \mathrm{mmol} / \mathrm{l}$ as per contemporary guidelines. For the women with more than one delivery during the study period, the first pregnancy with an abnormal OGTT result was selected as the index pregnancy. The women with GDM were seen regularly in the Prenatal Outpatient Clinic in Kuopio University Hospital and they received dietary advice, regular blood glucose monitoring and insulin treatment when necessary. The hospital register included data on maternal characteristics and pregnancy risk factors, complications, pregnancy outcome, and on the neonatal period of the offspring. The women with overt T2DM at the time of pregnancy or type 1 diabetes mellitus (T1DM) diagnosed after the index pregnancy, and those with a multiple pregnancy were excluded to eliminate confounding factors.

\section{The follow-up study}

The participants were recruited to the follow-up study between 2006 and 2009. The women underwent laboratory tests, body composition and blood pressure measurements, and answered questionnaires concerning their family history and health behavior. All participants underwent a 2-h OGTT (75 g of glucose). MetS was diagnosed by waist circumference $\geq 80 \mathrm{~cm}$, and at least two of the following four criteria in accordance with the International Diabetes Federation (IDF) 2005 criteria [18]: blood pressure $\geq 130 / 85 \mathrm{mmHg}$, fasting plasma glucose $\geq 5.6 \mathrm{mmol} / \mathrm{l}$, serum triglycerides $\geq 1.7 \mathrm{mmol} / \mathrm{l}$, and HDL cholesterol $\leq 1.29 \mathrm{mmol} / \mathrm{l}$. These criteria were selected since they are similar to the current care guidelines of MetS in Finland. The women using medication for hyperglycemia, hypertension or dyslipidemia were included in the analysis for the components of MetS.

Height was measured to the nearest $0.5 \mathrm{~cm}$ and weight to the nearest $0.1 \mathrm{~kg}$. Body mass index (BMI) was calculated as weight $(\mathrm{kg})$ divided by the height $(\mathrm{m})$ squared. Waist circumference (at the midpoint between the lateral iliac crest and the lowest rib) was measured to the nearest $0.5 \mathrm{~cm}$.

\section{Laboratory determinations}

Plasma glucose was measured by an enzymatic hexokinase photometric assay (Konelab Systems reagents; Thermo Fischer Scientific, Vantaa, Finland). LDL-cholesterol, HDLcholesterol and total triglycerides were measured by enzymatic colorimetric tests (Konelab Systems reagents).

\section{Statistical analyses}

The statistical analyses were conducted using SPSS version 23 (SPSS Inc., Chicago, IL). $P<0.05$ was considered statistically significant. The results were given as the mean \pm SD or number of cases and percentages. Statistical differences in categorical variables between the study and comparison groups were evaluated using the $x^{2}$ test. Anthropometric and biochemical continuous variables were analyzed using Student's t-test, and log-transformed variables were used to correct for their skewed distribution when appropriate. Since the diagnosis of GDM was based on slightly different criteria depending on the origin of the blood during the data collection, a correlation coefficient was used to convert all values to correspond venous plasma levels. The correlation coefficient was based on the information from the Department of Clinical Chemistry at Kuopio University Hospital.

This study was approved by the local Ethics Committee of the Kuopio University Hospital in accordance with the Helsinki Declaration.

\section{Results}

The clinical characteristics of the study groups stratified according to the birth weight of the offspring in index 
pregnancy and at the follow-up are shown in Table 1. Women with GDM were older in both birth weight categories as compared to controls during the index pregnancy. Women in both GDM groups and the women without GDM but with an LGA delivery were of higher weight and more frequently multiparous than the controls. Women with LGA infants had more frequently a history of prior child's birth weight over $4000 \mathrm{~g}$ as compared to than those with AGA offspring. Furthermore, women with GDM and an LGA delivery had more often a prior spontaneous abortion. The study groups did not differ in the rate of prior cesarean section. The incidence of pre-eclampsia was higher in women with GDM. No significant differences were observed in gestational age at birth between the study groups.

At the time of the follow-up study, the women with GDM in both birth weight categories had shorter follow-up time. However, no difference in the mean age of the women was observed between the study groups.
The women with GDM and the ones without GDM but with an LGA delivery were of higher weight than the controls, although the study groups did not differ in weight gain during the follow-up time (Table 1).

The comparison of cardiovascular and metabolic parameters of the study groups at follow-up is shown in Table 2. The women with GDM in both birth weight groups and the women without GDM with an LGA delivery had significantly higher waist circumference than the control group; approximately $80 \%$ of the women in those three groups reached the $80 \mathrm{~cm}$ waist circumference limit. Both GDM groups had significantly lower HDL levels and higher fasting plasma glucose than the control group, with approximately $50 \%$ of the women with GDM exceeding the limit $5.6 \mathrm{mmol} / \mathrm{l}$ at the follow-up visit. The mean triglyceride levels were higher in women with GDM in both birth weight categories. However, the study groups did not differ significantly concerning the triglyceride level over $1.7 \mathrm{mmol} / \mathrm{l} \mathrm{re}$ quired for MetS criterion. No significant differences

Table 1 Clinical characteristics of the controls and GDM subjects in index pregnancy and at the follow-up study stratified according to the offspring's birth weight

\begin{tabular}{|c|c|c|c|c|}
\hline \multirow[t]{3}{*}{ Offspring's birth weight } & \multicolumn{2}{|c|}{$\begin{array}{l}\text { AGA (10-90th percentile) } \\
\text { Mean } \pm \text { SD or } \%\end{array}$} & \multicolumn{2}{|c|}{$\begin{array}{l}\text { LGA (>90th percentile) } \\
\text { Mean } \pm \text { SD or } \%\end{array}$} \\
\hline & No GDM & GDM & No GDM & GDM \\
\hline & (Controls) & (Group 1) & (Group 2) & (Group 3) \\
\hline Number of subjects & 286 & 376 & 48 & 68 \\
\hline \multicolumn{5}{|l|}{ At the index pregnancy } \\
\hline Age (yrs) & $29.5 \pm 5.4$ & $31.8 \pm 6.0^{* *}$ & $30.6 \pm 5.0$ & $32.6 \pm 6.3^{* *}$ \\
\hline Primiparity (\%) & 53.0 & $35.0^{* *}$ & $34.1^{*}$ & $22.2^{* *}$ \\
\hline Pre-pregnancy BMI (kg/m²) & $23.8 \pm 3.8$ & $26.4 \pm 5.0^{* *}$ & $25.7 \pm 3.5^{*}$ & $26.7 \pm 4.1^{* *}$ \\
\hline Family history of diabetes (\%) & 69.4 & $81.4^{* *}$ & 75.0 & 80.9 \\
\hline Prior child's birth weight > $4000 \mathrm{~g}(\%)$ & 25.4 & 25.6 & $43.8^{*}$ & $60.4^{* *}$ \\
\hline Prior spontaneous abortion (\%) & 16.8 & 19.9 & 18.8 & $35.3^{*}$ \\
\hline Prior cesarean section (\%) & 5.9 & 9.6 & 16.7 & 7.4 \\
\hline Gestational age (d) & $280 \pm 11$ & $279 \pm 9$ & $279 \pm 11$ & $278 \pm 8$ \\
\hline Pre-eclampsia (\%) & 1.4 & $5.3^{*}$ & 2.1 & $5.9^{*}$ \\
\hline Birth weight (g) & $3595 \pm 385$ & $3596 \pm 406$ & $4365 \pm 424^{* *}$ & $4421 \pm 370^{* *}$ \\
\hline Placental-fetal mass ratio (\%) & $17.1 \pm 3.0$ & $17.5 \pm 3.0$ & $20.6 \pm 15.8^{* *}$ & $17.9 \pm 2.5^{*}$ \\
\hline Low Apgar score 1 min $<7(\%)$ & 1.7 & $5.9^{*}$ & 6.3 & 4.4 \\
\hline \multicolumn{5}{|l|}{ At the follow-up study } \\
\hline Follow-up time (yrs) & $8.5 \pm 5.5$ & $5.3 \pm 4.3^{* *}$ & $7.4 \pm 5.4$ & $6.2 \pm 4.9^{*}$ \\
\hline Age at follow-up (yrs) & $38.4 \pm 6.4$ & $37.4 \pm 7.2$ & $38.3 \pm 5.8$ & $39.1 \pm 7.5$ \\
\hline BMI $\left(\mathrm{kg} / \mathrm{m}^{2}\right)$ & $26.5 \pm 4.9$ & $28.3 \pm 5.7^{* *}$ & $27.9 \pm 4.8^{*}$ & $29.2 \pm 4.9^{* *}$ \\
\hline $\begin{array}{l}\text { Weight gain during the follow-up } \\
\text { time }(\mathrm{kg})\end{array}$ & $5.7 \pm 7.6$ & $3.6 \pm 7.9$ & $4.3 \pm 7.6$ & $5.6 \pm 9.3$ \\
\hline
\end{tabular}

GDM gestational diabetes mellitus, $B M I$ body mass index, $A G A$ appropriate for gestational age, $L G A$ large for gestational age

All groups compared to controls separately

${ }^{*} p<0.05$

${ }^{* *} p<.0001$ 
Table 2 The components of the metabolic syndrome (MetS) in the study subjects at the follow-up study stratified according to the offspring's birth weight

\begin{tabular}{|c|c|c|c|c|}
\hline \multirow[t]{3}{*}{ Offspring's birth weight } & \multicolumn{2}{|c|}{$\begin{array}{l}\text { AGA (10-90th percentile) } \\
\text { Mean } \pm \text { SD or } \%\end{array}$} & \multicolumn{2}{|c|}{$\begin{array}{l}\text { LGA (>90th percentile) } \\
\text { Mean } \pm \text { SD or } \%\end{array}$} \\
\hline & No GDM & GDM & No GDM & GDM \\
\hline & (Controls) & (Group 1) & (Group 2) & (Group 3) \\
\hline Number of subjects & 286 & 376 & 48 & 68 \\
\hline Waist circumference $(\mathrm{cm})$ & $85.3 \pm 11.6$ & $91.0 \pm 13.8^{* *}$ & $88.8 \pm 10.8^{*}$ & $94.2 \pm 12.9^{* *}$ \\
\hline Waist circumference $\geq 80 \mathrm{~cm}(\%)$ & 64.0 & $77.6^{* *}$ & $81.3^{*}$ & $89.7^{* *}$ \\
\hline Fasting glucose (mmol/l) & $5.3 \pm 0.4$ & $5.6 \pm 0.8^{* *}$ & $5.4 \pm 0.4$ & $5.8 \pm 0.8^{* *}$ \\
\hline Fasting glucose $\geq 5.6$ mmol/l (\%) & 25.2 & $46.8^{* *}$ & 29.2 & $58.8^{* *}$ \\
\hline Triglycerides (mmol/l) & $1.0 \pm 0.6$ & $1.1 \pm 0.6^{*}$ & $0.9 \pm 0.4$ & $1.2 \pm 0.5^{*}$ \\
\hline Triglycerides $\geq 1,70 \mathrm{mmol} / \mathrm{l}(\%)$ & 10.6 & 15.2 & 4.2 & 16.2 \\
\hline HDL cholestrol (mmol/l) & $1.5 \pm 0.4$ & $1.4 \pm 0.4^{* *}$ & $1.6 \pm 0.3$ & $1.3 \pm 0.3^{* *}$ \\
\hline HDL cholestrol < 1.29 mmol// (\%) & 28.3 & $44.1^{* *}$ & 20.8 & $57.4^{* *}$ \\
\hline Systolic pressure (mmHg) & $122.5 \pm 14.3$ & $125.8 \pm 14.1^{*}$ & $121.4 \pm 10.1$ & $128.4 \pm 14.2^{*}$ \\
\hline Diastolic pressure $(\mathrm{mmHg})$ & $78.3 \pm 9.6$ & $79.3 \pm 9.2$ & $77.5 \pm 7.5$ & $81.3 \pm 10.0^{*}$ \\
\hline Blood pressure $\geq 130 / \geq 85 \mathrm{mmHg}(\%)$ & 34.3 & 38.8 & 27.1 & 45.6 \\
\hline Metabolic syndrome (IDF) (\%) & 24.5 & $43.6^{* *}$ & 18.8 & $54.4^{* *}$ \\
\hline
\end{tabular}

GDM gestational diabetes mellitus, $B M I$ body mass index, $A G A$ appropriate for gestational age, $L G A$ large for gestational age All groups compared to controls separately

${ }^{*} p<0.05$

$* * p<.0001$

were observed in total blood pressure between the study groups, even though the mean systolic blood pressure in both GDM groups and diastolic pressure in the women with GDM and LGA infants was significantly higher as compared to controls (Table 2).

The incidence of MetS at the follow-up study stratified with the birth weight of the offspring is illustrated in Fig. 1.
The incidence of MetS was higher in women with GDM and an LGA (54.4\%) than an AGA delivery (43.6\%). Furthermore, the incidence of MetS in LGA study groups was three times higher in women with GDM as compared to the normoglycemic women. However, the incidence of MetS did not differ significantly in the non-GDM group between the AGA (24.5\%) and LGA (18.8\%) groups.

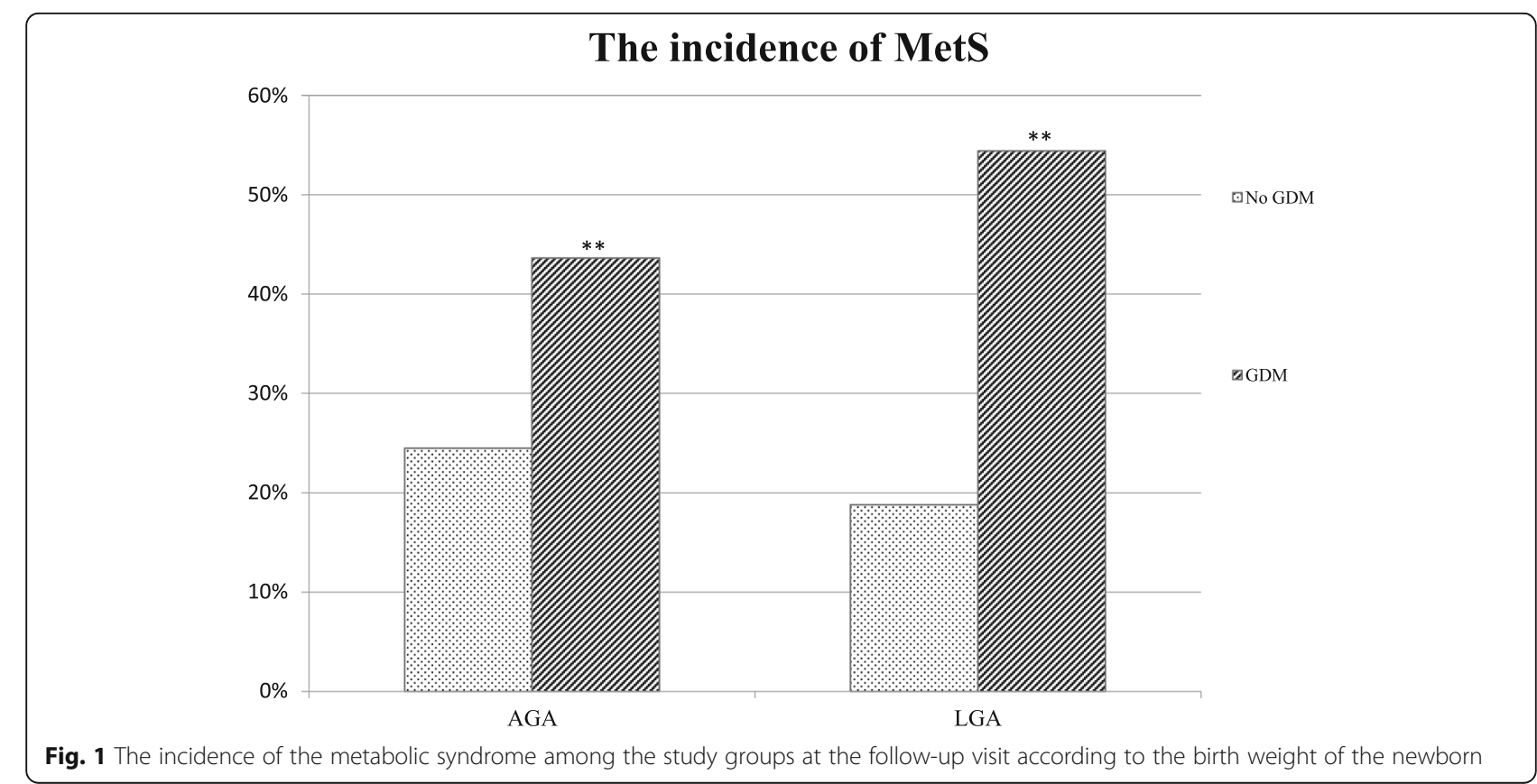




\section{Discussion}

Our long-term study indicated that after an LGA delivery, the incidence of MetS is three times higher in women with GDM compared to those without GDM. Increased waist circumference was the only component of MetS in the non-GDM LGA group that was more prevalent than in the control group. Among the women with GDM, the limits of MetS in fasting glucose and HDL cholesterol were broken more often than in the control group. Overall, LGA delivery alone did not predict future MetS in women with normal glucose tolerance during pregnancy. In contrast, delivery of an LGA infant in women with GDM predicts future risk of MetS and thus risk for future cardiovascular disease.

Previously, only few studies have focused on the components of MetS separately after an LGA delivery without GDM. In our study, the mean waist circumference was significantly greater in women with GDM at follow-up in both birth weight categories. In addition, $81.3 \%$ of the women with a prior LGA delivery without GDM exceeded $80 \mathrm{~cm}$ waist circumference limit fulfilling the compulsory criterion of MetS. An explanation for this could be high pre-pregnancy BMI in this study group and genetic susceptibility to such body composition. These results are in agreement with a previous 18-years follow-up study showing that waist circumference and fasting glucose were the only significant components of MetS in mothers with LGA infants with or without GDM during their pregnancies [8]. In agreement with our results, no difference was observed in waist circumference in women with macrosomic $(>4 \mathrm{~kg})$ or stillborn newborns as compared to ageand BMI-matched women without macrosomic deliveries in a 9-year follow-up study in women without previous GDM [19].

The development of dysglycemia and type 2 diabetes in women with a background of GDM supported by a large body of evidence $[1,2]$. In accordance, a higher percentage of fasting glucose $>5.6 \mathrm{mmol} / \mathrm{l}$ was detected at follow-up in both GDM groups as compared to controls regardless of the birth weight category. However, no significant difference in fasting glucose was observed in women with a previous LGA delivery without GDM compared to controls. This is in agreement with a previous 2-year follow-up study, where fasting glucose levels did not differ between the non-GDM women with previous LGA and AGA deliveries [20]. Moreover, a 9-year follow-up study did not find any differences in fasting glucose concentrations in women with and without previous macrosomic newborns with absence of GDM during pregnancy [19].

In our study, LGA delivery without previous GDM did not predict later dyslipidemia as compared to the controls. Mean HDL cholesterol levels were lower and triglycerides slightly higher in women with GDM in both birth weight categories. However, concerning the lipid components of MetS, only low HDL cholesterol was more prevalent in the GDM groups than in controls. In agreement with our findings, a study performed 2 years after pregnancy revealed that no significant differences were observed between 18 women with LGA infants and 18 women with AGA infants with respect to lipids [20]. Correspondingly, a 9-year follow-up study demonstrated no differences in the incidence of dyslipidemia between 570 women with a history of macrosomia or stillbirth without GDM compared to age- and BMI-matched controls [19]. In contrast to these reports, a study of 48 women with previous birth of large infants and without glucosuria during pregnancy demonstrated that after 20-27 years postpartum these women had significantly lower concentration of HDL-cholesterol compared to age-, parity- and BMI-matched controls with birth weight $<4500 \mathrm{~g}$ [21]. Further, a study of 332 women with a prior LGA delivery reported lower HDL-cholesterol levels than in 2630 women with an appropriate-for-gestational-age (AGA) newborns in an age-adjusted model 18 years after pregnancy [8]. However, when adjusted for confounders the statistical significance was lost.

No studies have reported on the prevalence of the MetS high blood pressure criterion $(\geq 130 / \geq 85 \mathrm{mmHg})$ after an LGA delivery. We found no differences in this prevalence between the study groups. In agreement with our results, a prior macrosomic or LGA delivery in women without GDM did not predict later increased systolic or diastolic blood pressure in two-previous follow-up studies [19, 21].

Although some previous research has been carried out on components of MetS after an LGA delivery, the overall incidence of MetS has not been known. Our results show that $54.4 \%$ of women with GDM and an LGA delivery developed incident MetS during the follow-up, as compared to $43.6 \%$ in the AGA group. Interestingly, in women without GDM, the incidence of MetS was not higher with a previous LGA delivery as compared to the group with AGA delivery even though maternal BMI was higher in the LGA group. In this study, a considerable part of the women without GDM were overweight $\left(\mathrm{BMI} \geq 25 \mathrm{~kg} / \mathrm{m}^{2}\right.$ ) in pre-pregnancy: $28.7 \%$ in the AGA and $51.1 \%$ in the LGA group (data not shown) as a result of risk-based screening for GDM. Therefore, it could be assumed that an LGA delivery in women without GDM is not predictive for later metabolic risk factors and MetS. Similarly, no association between an LGA delivery with the calculated 10-year CVD risk after adjustment for confounders was found in another study [8].

The strengths of the current study included the long-term follow-up of a well-characterized cohort of women, and the similar treatment received by all participants with GDM during pregnancy. It should be noted 
that in the present study, the GDM criteria in years 19892008 were tight especially regarding the fasting glucose value in OGTT. Thus, some women with GDM who would not be diagnosed with GDM using the current criteria were included as GDM women. This analysis has concentrated on women who were chosen from an obstetric population with risk factors for GDM potentially causing some selection bias. In addition, the study setting was cross-sectional at the time of follow-up OGTT, not longitudinal which would have been optimal to standardize the protocol. Notwithstanding its limitations, this study does suggest that even though all subjects have GDM risk factors, an LGA delivery does not predict later MetS in women without GDM.

In conclusion, the women without GDM were at a lower risk than those with GDM for MetS even with an LGA delivery. This probably reflects good maternal vascular health and its effects on birth weight. In contrast, women with GDM and a previous LGA delivery should be considered as a high-risk target group for prevention of future MetS and CVD.

\section{Conclusion}

In summary, an LGA delivery without GDM was not significantly associated with future maternal MetS risk in the mean follow-up time of 7.3 years. High offspring birth weight in this group is likely to be related to maternal vascular health and genetic factors. In contrast, women with GDM who have had an LGA delivery should have a stringent follow-up after pregnancy to reduce the risk of future MetS and enhance women's cardiovascular health.

\section{Abbreviations}

AGA: Appropriate for gestational age; BMI: Body mass index; CVD: Cardiovascular diseases; GDM: Gestational diabetes; HDL: High density lipoprotein; IDF: International Diabetes Federation; LDL: Low density lipoprotein; LGA: Large for gestational age; MetS: Metabolic syndrome; OGTT: Oral glucose tolerance test; T1DM: Type 1 diabetes mellitus; T2DM: Type 2 diabetes

\section{Acknowledgements}

We would like to acknowledge all the patients who participated in this study. For statistical guidance and help, we want to thank Olavi Kauhanen and Tuomas Selander, Kuopio university hospital.

\section{Authors' contribution \\ $\mathrm{SH}$ and $\mathrm{HHu}$ had the original idea for this study and collected the data. $\mathrm{HHa}$ analysed the data, interpreted the results, and wrote this article. SH provided supervision and advice on the data available, interpretation of the results, and revised article critically. $\mathrm{HHu}, \mathrm{RV}$ and $\mathrm{HC}$ provided supervision and revised article critically. All authors read and approved the final version of the article.}

\section{Funding}

The study was supported by Kuopio University Hospital VTR-grants, Finnish Cultural Foundation, North Savo Regional fund and The Finnish Medical Foundation. The funders had no role in study design, data collection and analysis, decision to publish, or preparation of the manuscript.

\section{Availability of data and materials}

The datasets analysed during the current study are neither publicly nor upon request available due to national regulations and them containing

information that could compromise participant privacy.

\section{Ethics approval and consent to participate}

This study was approved by the local Ethics Committee of the Kuopio University Hospital in accordance with the Helsinki Declaration. All participants gave a written informed consent.

\section{Consent for publication}

Not applicable.

\section{Competing interests}

The authors declare that they have no competing interests.

\section{Publisher's Note}

Springer Nature remains neutral with regard to jurisdictional claims in published maps and institutional affiliations.

\section{Author details}

${ }^{1}$ Department of Obstetrics and Gynecology, Kuopio University Hospital, Puijonlaaksontie 2, P.O.B 100, 70029 KYS, Kuopio, Finland. ${ }^{2}$ Institute of Clinical Medicine, School of Medicine, University of Eastern Finland, P.O.B 1627, 70211 Kuopio, Finland. ${ }^{3}$ Department of Pediatrics, Kuopio University Hospital, P.O.B 100, 70029 KYS, Kuopio, Finland. ${ }^{4}$ Department of Medicine, Helsinki University Hospital, Jorvi Hospital, P.O.B 800, 00029 HUS, Helsinki, Finland. ${ }^{5}$ Faculty of Medicine, Center for Life Course Epidemiology and Systems Medicine, University of Oulu, P.O.B 8000, 90014 Oulu, Finland. ${ }^{6}$ Department of Pediatrics, University of Eastern Finland, P.O.B 1627, 70211 Kuopio, Finland. ${ }^{7}$ Department of Obstetrics and Gynecology, Helsinki University Central Hospital, P.O.B 140, 00029 HUS, Helsinki, Finland. ${ }^{8}$ Department of Obstetrics and Gynecology, University of Helsinki, P.O.B 3, 00014 Helsinki, Finland.

Received: 13 September 2017 Accepted: 31 July 2018

Published online: 10 August 2018

\section{References}

1. Bellamy L, Casas JP, Hingorani AD, Williams D. Type 2 diabetes mellitus after gestational diabetes: a systematic review and meta-analysis. Lancet. 2009; 373(9677):1773-9.

2. Kim C, Newton KM, Knopp RH. Gestational diabetes and the incidence of type 2 diabetes: a systematic review. Diabetes Care. 2002;25(10):1862-8.

3. Hakkarainen $\mathrm{H}$, Huopio $\mathrm{H}$, Cederberg $\mathrm{H}$, Paakkonen $\mathrm{M}$, Voutilainen $\mathrm{R}$, Heinonen S. Post-challenge glycemia during pregnancy as a marker of future risk of type 2 diabetes: a prospective cohort study. Gynecol Endocrinol. 2015:31(7):573-7.

4. Xu Y, Shen S, Sun L, Yang H, Jin B, Cao X. Metabolic syndrome risk after gestational diabetes: a systematic review and meta-analysis. PLoS One. 2014; 9(1):e87863

5. Hakkarainen $\mathrm{H}$, Huopio $\mathrm{H}$, Cederberg $\mathrm{H}$, Paakkonen $\mathrm{M}$, Voutilainen $\mathrm{R}$, Heinonen $\mathrm{S}$. The risk of metabolic syndrome in women with previous GDM in a long-term follow-up. Gynecol Endocrinol. 2016;32:920-5.

6. Shah BR, Retnakaran R, Booth GL. Increased risk of cardiovascular disease in young women following gestational diabetes mellitus. Diabetes Care. 2008; 31(8):1668-9.

7. Retnakaran R, Shah BR. Mild glucose intolerance in pregnancy and risk of cardiovascular disease: a population-based cohort study. CMAJ. 2009;181(6-7):371-6.

8. Fraser A, Nelson SM, Macdonald-Wallis C, Cherry L, Butler E, Sattar N, et al. Associations of pregnancy complications with calculated cardiovascular disease risk and cardiovascular risk factors in middle age: the Avon longitudinal study of parents and children. Circulation. 2012;125(11):1367-80.

9. Alberti KG, Eckel RH, Grundy SM, Zimmet PZ, Cleeman JI, Donato KA, et al. Harmonizing the metabolic syndrome: a joint interim statement of the international diabetes federation task force on epidemiology and prevention; National Heart, Lung, and Blood Institute; American Heart Association; world heart federation; international atherosclerosis society; and International Association for the Study of obesity. Circulation. 2009:120(16): $1640-5$. 
10. Pedersen J. Diabetes and Pregnancy: Blood Sugar of Newborn Infants. [dissertation]. Copenhagen, Denmark, Danish Science Press.; 1952.

11. Berntorp K, Anderberg E, Claesson R, Ignell C, Kallen K. The relative importance of maternal body mass index and glucose levels for prediction of large-for-gestational-age births. BMC Pregnancy Childbirth. 2015;15:280.

12. Yu Z, Han S, Zhu J, Sun X, Ji C, Guo X. Pre-pregnancy body mass index in relation to infant birth weight and offspring overweight/obesity: a systematic review and meta-analysis. PLoS One. 2013;8(4):e61627.

13. Ludwig DS, Currie J. The association between pregnancy weight gain and birthweight: a within-family comparison. Lancet. 2010;376(9745):984-90.

14. Kitajima M, Oka S, Yasuhi I, Fukuda M, Rii Y, Ishimaru T. Maternal serum triglyceride at 24-32 weeks' gestation and newborn weight in nondiabetic women with positive diabetic screens. Obstet Gynecol. 2001;97(5 Pt 1):776-80.

15. Clausen T, Burski TK, Oyen N, Godang K, Bollerslev J, Henriksen T. Maternal anthropometric and metabolic factors in the first half of pregnancy and risk of neonatal macrosomia in term pregnancies. A prospective study. Eur J Endocrinol. 2005;153(6):887-94.

16. Boney CM, Verma A, Tucker R, Vohr BR. Metabolic syndrome in childhood: association with birth weight, maternal obesity, and gestational diabetes mellitus. Pediatrics. 2005;115(3):e290-6.

17. Sankilampi U, Hannila ML, Saari A, Gissler M, Dunkel L. New population-based references for birth weight, length, and head circumference in singletons and twins from 23 to 43 gestation weeks. Ann Med. 2013;45(5-6):446-54.

18. Alberti KG, Zimmet P, Shaw J, IDF Epidemiology Task Force Consensus Group. The metabolic syndrome--a new worldwide definition. Lancet. 2005; 366(9491):1059-62

19. Tehrani FR, Hashemi S, Hasheminia M, Azizi F. Follow-up of women with gestational diabetes in the Tehran lipid and glucose study (TLGS): a population-based cohort study. J Obstet Gynaecol Res. 2012;38(4):698-704.

20. Moses R, Davis W, Rodgers D, Meyer B, Calvert D. The metabolic profile of glucose tolerant women who have had large for gestational age babies. Aust N Z J Obstet Gynaecol. 1997;37(2):177-80.

21. Spjuth J, Larsson G, Nilsson-Ehle P, Schersten B, Astedt B. Can the birth of a large infant predict risk for atherosclerotic vascular disease in the mother? Diabetologia. 1993;36(2):117-20.

Ready to submit your research? Choose BMC and benefit from:

- fast, convenient online submission

- thorough peer review by experienced researchers in your field

- rapid publication on acceptance

- support for research data, including large and complex data types

- gold Open Access which fosters wider collaboration and increased citations

- maximum visibility for your research: over $100 \mathrm{M}$ website views per year

At $\mathrm{BMC}$, research is always in progress.

Learn more biomedcentral.com/submissions 